\title{
Design of Teaching Management System of University Experiment Based on Cloud Computing
}

\author{
LianminCui \\ University of Shanghai for Science and Technology, Lab Management and Service Center, \\ Shanghai, China \\ cuilianmin@163.com
}

\begin{abstract}
In order to promote the informationization development of experiment course teaching in university, it is necessary to integrate the software resources and the experimental teaching resources scattered in various experimental center and to realize the sharing of resources and effective use. This paper designed the university experiment teaching management system based on cloud Computing technology and advantages, put forward the management system of university experimental teaching requirements and design the university curriculum management system architecture based on cloud computing. It provides a new way for improving the level of teaching management of experimental course.
\end{abstract}

Keywords: Cloud computing, Experiment course, Management system.

\section{基于云计算的大学实验教学管理系统设计}

\section{崔连敏}

上海理工大学实验室管理与服务中心, 上海, 中国 cuilianmin@163.com

摘要: 为了促进大学实验课程教学信息化发 展, 整合分散在各个实验中心的软件资源及 实验教学资源, 实现资源共享和有效利用, 本文设计了基于云计算的大学实验教学管理 系统。在研究云计算相关技术及优势的基础 上, 提出了大学实验课程管理系统的需求, 设计了基于云计算的大学实验课程管理系统 的架构, 为提高实验课程教学管理水平提供 了新方法。

关键词: 云计算; 大学实验课程; 教学管理 系统;

\section{1 引言}

大学实验课程包括计划性实验、开放式 自主性实验、提高性综合实验和大学生创新
性实验等, 主要培养学生在专业基础学习阶 段账务扎实的理论基础和实验动手能力。教 育信息化是指在教育管理、教学和科研等领 域全面深入地运用现代信息技术来促进教育 改革与发展的过程。随着高校教育信息技术 的快速发展, 诸多问题也开始涌现出来。例 如当前传统的实验教学管理系统面临资源重 复投入建设, 各个应用系统之间接口不一致, 系统维护成本高, 实验数据和资源无法共享 等问题[1]。

云计算技术是一项被认为是紧接着个人 电脑与互联网技术后, 又一项划时代的信息 技术革命性的新技术，在诸多领域都得到了 基本的应用。为了减少硬件资源投入和维护 成本, 建立统一开发的大学实验教学管理系 
统, 本文基于云计算虚拟化、高扩展性和资 源利用率高等特点, 根据实际需要设计了基 于云计算的大学实验课程教学管理系统。

\section{2 云计算}

云计算是分布计算、并行计算、网络存 储、虚拟化、负载均衡和热备份几余等传统 计算机和网络技术发展融合的产物。云计算 致力于解决网络平台的通讯、存储和资源利 用等问题 [2]。

自 2006 年 Google 公司首席执行官首次 提出云计算的概念以来, 云计算技术发展十 分迅速。目前Google、IBM、亚马逊、微软 等国际化大公司纷纷推出了自己的云计算平 台, 国内的华为、阿里巴巴等也相继启动了 云计算的项目。典型的云计算架构如图 1 所 示, 分为基础设施层、平台层和应用层。基 础设施层主要提供基础设施即服务 ( IssS), 平台层提供平台即服务 (Paas), 应用层提供 软件即服务（SaaS） [3][4]。

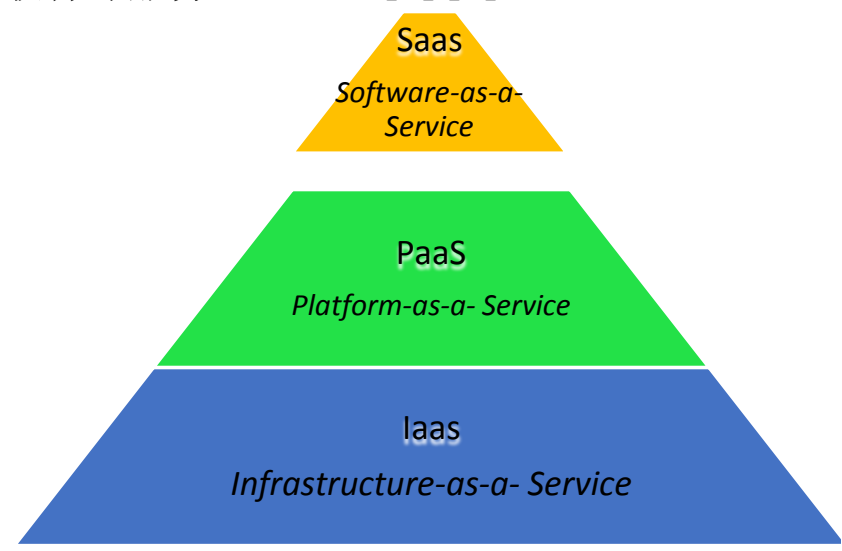

图 1 云计算基础架构

在整合本校目前各实验中心现有软硬件 资源基础上建设基于云计算的实验课程教学 管理系统, 从资源共享方面能够实现软硬件 集中部署、统建共用避免重复投资。系统可 以按照SOA松耦合方式建设, 系统模块之间通 过标准协议实验相互调用和消息交换, 有利 于各实验中心实现数据共享 $[5]$ 。在管理工作 方面按照统一标准开发部署的管理系统, 能 够统一制定各个实验中心工作流程和管理规 范标准化, 大大节省了实验室及实验教学的 管理成本。

\section{3 基于云计算的大学实验课程教学管理系统 设计}

\section{1 系统需求分析}

本文主要针对当前普通大学实验课程教 学管理系统设计存在的问题, 结合云计算技 术的优点设计了基于云计算的大学实验课程 教学管理系统, 主要实现高校实验课程教学 的云管理, 推动大学实验课程教学管理创新, 为高校更好的进行实验课程教学奠定基础。

系统主要有教师、学生、教学管理员和 系统管理员等用户组成。系统设计需要满足 各类用户的需求, 要求能够实现对实验室、 实验课程以及实验教学的管理, 教学资源的 整合共享和有效利用, 可以根据不同的用户 设置不同的用户权限和访问空间，能够满足 师生互动交流的功能, 能够生成相关统计分 析数据和报表。同时为了使整个系统良好的 运行, 系统还需要提供日常运行日志和错误 日志、数据备份及管理等功能。

为了提供较好的用户体验, 还需要考虑 系统的性能指标，根据本校的实际情况设计 性能指标为日均访问量 $>350$ 人次、平均响应 时间 $<10$ 秒、最大响应时间 $<20$ 秒和并发用户 数 $>20$ 人等。另外由于目前移动互联网发展 十分迅速, 系统还需要支持移动设备如 Android 设备如三星、小米、华为等热门机 型、IOS 设备如 IPhone $5 / 5 \mathrm{~s} / 6 / 6 \mathrm{P} 1 \mathrm{us}$ 等、平 板电脑如 IPad4、IPAD Air 等访问, 要求在 移动设备能够实现全屏或匹配浏览器的自适 应汶览显示, 可适应横屏和坚屏汶览的需要, 同时满足智能手机平台下的主流手机汶览器 适配。系统还需要为后续开发扩展留有余地。

\section{2 系统架构设计}

基于云计算大学实验课程教学管理系统 通过服务器虚拟化和分布式存储等技术, 实 现各个实验中心软硬件资源和实验教学资源 协同工作，完成教学资源整合、应用系统集 成和资源数据共享。参照云计算基础架构, 设计了基于云计算的实验课程教学管理系统 的核心架构如图 2 所示，分成基础设施层、 平台管理层、应用服务层。

基础设施层主要有服务器 X86 服务器、 小型机服务器、数据库、网络设备等组成, 是整个系统的基础。通过使用 VMware 虚拟化 
技术在物理服务器上生成多个虚拟机, 实现 对所有硬件资源虚拟化管理, 为用户和其它 各层提供其所需的计算、存储和带宽等功能, 不仅节省了硬件资源的购置成本, 而且使得 系统的运维变得更加方便。

平台管理层主要通过互联网为实验教学 管理系统提供整套开发、运行和应用软件的 支撑平台。主要提供应用管理、工作流管理、 数据交换管理、搜索服务、信息安全管理、 用户权限管理、用户登录认证服务等功能。
应用服务层主要集成了目前各实验中心 教学管理系统、实验室管理系统、实验课程 管理系统，同时为了促进师生的交流互动, 设计了师生互动系统, 包括师生即时沟通工 具、Emai1 以及 BBS 论坛等子模块。普通用 户可以使用户使用计算机、Android 和 IOS 移动终端以及平板电脑等设备, 通过互联网 统一入口登陆到实验课程教学管理系统, 根 据设置的用户权限获取相应的资源。

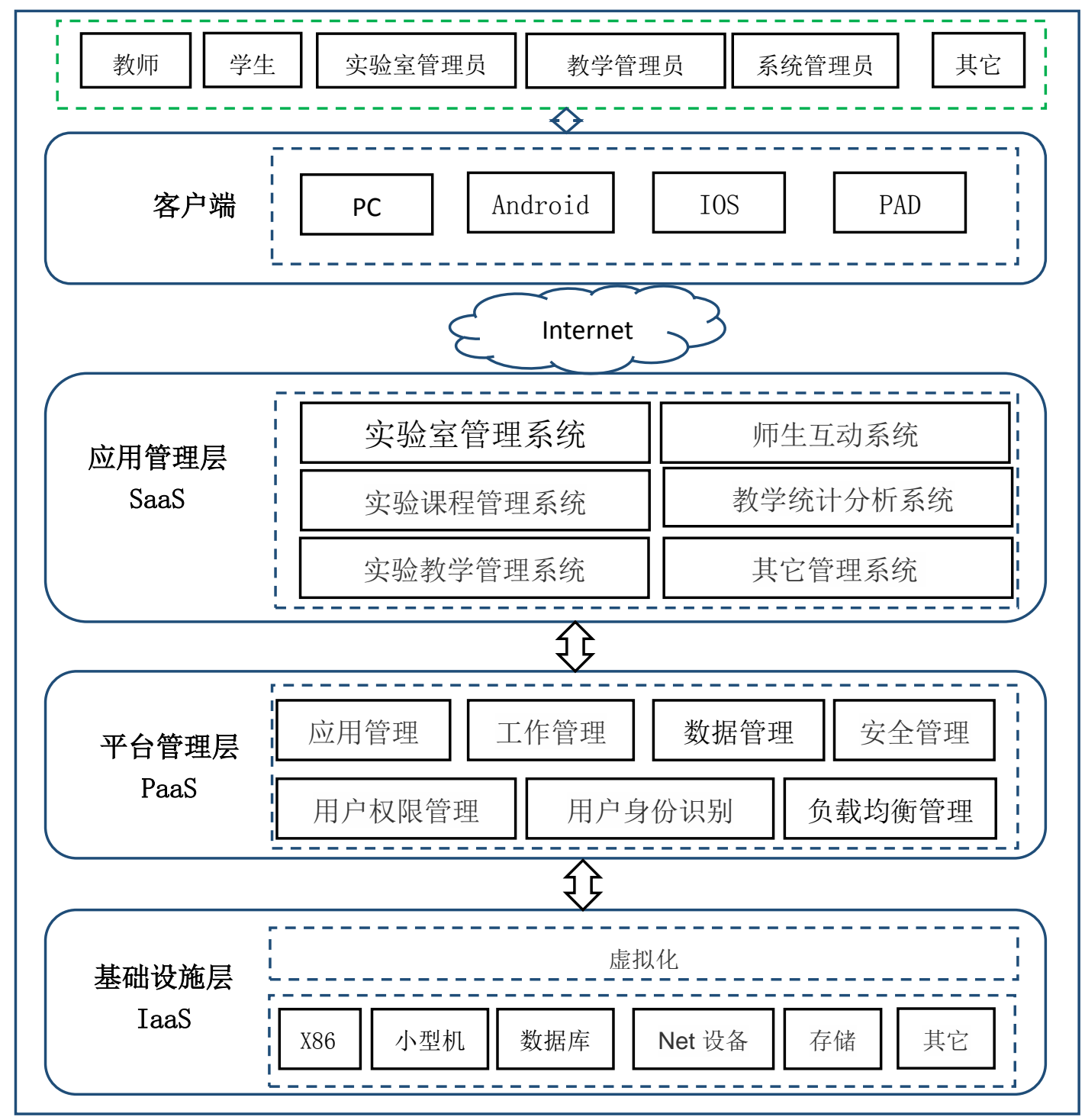

图 2 基于云计算的实验教学管理系统架构图

\section{3 系统实现功能}

基于云计算的实验课程教学管理系统具 备高性能的计算资源以及海量的存储资源, 主要实现以下几方面的功能。

实验室管理模块主要提供实验室管理与 服务中心下属各实验中心简介、机构设置、
师资队伍、工作动态等相关信息展示及维护; 实验室规章制度发布; 各实验中心通讯信息 添加与修改功能; 学生通过该系统查看开发 实验室预定情况, 也可以选择预定处于空闲 状态开放实验室，管理人员及教师可以统计 分析各实验室的预定情况等功能。 
实验课程管理模块主要提供各实验中心 所有的大学实验课程介绍; 各实验课程代码、 课程名称、学分、学时、建议修读学期、考 核方式等信息增删及修改功能; 教师可以将 实验课程资料上传至云服务器, 学生可以登 录到相应链接下载查询相关资料等功能。

实验教学管理模块主要提供学生登录系 统选择选课修读学期; 教学管理人员根据实 际情况安排课程表并发布到系统中; 学生可 以登录到系统中预定实验位置; 学生可以记 录实验数据到系统中, 教师可以审阅学生实 验结果是否正确; 教师可以在系统中发布实 验作业, 学生完成实验作业后提交至该系统 中, 教师在线批阅立即反馈学生用户; 实验 在线考试等功能。虚拟实验课堂主要提供学 生可以根据各实验中心已录制的在线视频学 习实验内容; 实验虚拟仿真平台能够形象的 展示实验原理及过程, 提高教学质量。

实验教学质量评价模块主要为了提高实 验教学质量。实验室教学管理者根据学校相 关规章制度在, 制定教学评价标准。学生上 完每个实验后在系统中根据教学评价标准逐 项评价。教学督导委员会及实验教学指导委 员会成员也能根据督导及检查情况, 汇总结 果到系统中。

师生互动管理模块主要提供即时聊天功 能、电子邮件功能、论坛 BBS 功能, 教师在 线答疑功能等。

教学数据统计分析模块主要自动生成实 验室设备统计分析报告、学生学习过程统计 分析报告、教师教学质量统计分析报告、学 生考试成绩统计分析报告等功能。

系统管理模块主要提供用户权限管理设 置、系统运行日志查看、系统错误日志查看、 系统数据备份等功能。

\section{4 结束语}

本文将云计算引入大学实验课程教学管 理系统建设中, 旨在将高校实验室、实验课 程及实验教学管理各环节建立标准化的规范 流程, 实现了各实验中心的资源和数据共享, 为提高实验课程教学管理水平提供了新方法。

\section{致谢}

本文为上海青年教师资助计划项目 (1014204804)的阶段性成果之一。

\section{References}

[1] Wu yanqing, Design of a laboratory management information system based on cloud computing, Research and exploration in laboratory, vol.32, pp.291-296, 2013.

[2] Li Qiao, Zheng xiao, Research survey of cloud computing, computer science, Vol. 38, pp.32-37, 2011

[3] Luo Junzhou, Jin Jiahui, Song aibo and Dong Fang, Cloud computing: architecture and key technology, journal on communication, Vol.32, pp.3-21,2007

[4] Wang Lili, Gao Xincheng, Li Ruifang and Liu Jinyue, The design of teaching resource management platform based on cloud computing, Journal of Shaanxi University of technology(Nature science Edition), Vol.30,2014

[5] Ling Xiaodong, A review of SOA, Computer application and software, Vol.24, pp.122-12 